\title{
Effect of surface charges on the curvature moduli of a membrane
}

\author{
V. Kumaran \\ Department of Chemical Engineering, Indian Institute of Science, Bangalore 560 012, India
}

\begin{abstract}
The modification of the curvature moduli due to surface charges in lipid bilayers is analyzed using the nonlinear Poisson-Boltzmann equation for the relationship between the charge density and surface potential. An expansion in a small parameter $\epsilon$, which is the ratio of the Debye length and the radius of curvature, is used. At low charge densities, previous results obtained from the Debye-Huckel approximation are recovered. At high charge densities, the corrections to the mean and Gaussian curvature approach constant values. The total energy of curvature for a symmetrically charged membrane becomes negative when the charge density is increased beyond a critical value, indicating that the membrane spontaneously forms vesicles. An asymmetry in the charge densities on the two monolayers that form the bilayer results in a spontaneous curvature, and the radius of curvature could be large compared to the Debye length when the asymmetry is small. The case of adsorbed charges is also considered, where there is a reduction in the chemical energy when a charge is adsorbed on the surface. At low charge density, the mean and Gaussian curvature are equal in magnitude and opposite in sign to that for fixed charges, while at high charge density, the mean and Gaussian curvature approach values identical to that for a surface with fixed charges. Numerical calculations of the change in the curvature moduli with realistic parameter values indicate that these effects are likely to be of importance in the spontaneous formation of vesicles.
\end{abstract}

\section{INTRODUCTION}

Vesicles, which are lipid bilayers shaped in the form of spheres, are of interest in many practical applications, such as drug delivery systems. These are usually made under nonequilibrium conditions, because the bending energy for the formation of a vesicle of micron size is large compared to the thermal energy. However, some interesting experimental results [1] have revealed that stable vesicles could be formed at equilibrium if a mixture of lipids with surface charges of opposite signs are used. The relationship between the surface charge density and the curvature could also be of importance in biological systems. Two of the salient features of surfaces encountered in biological systems, such as cell membranes and organelles, are that they are soft and can undergo shape changes, and they have adsorbed charges. It is well known [2] that significant variations in charge distribution and the transmembrane potential of membranes coincide with shape changes.

Previous studies have examined the shape changes of biological membranes due to forces exerted by ion transport in proteins, due to the asymmetry of inclusions in the membrane and their phase separation on the surface, and other nonequilibrium processes [3]. Though most of the studies on biological membranes have examined fluctuations at thermal equilibrium [4], it has recently been realized that the forces generated on membranes by inclusions could play a crucial role in the structure and dynamics of membranes. Phase separation of the components of a membrane could also alter the shape [5]. However, it is expected that effects like headtail asymmetry would lead to structures with characteristic lengths of the same magnitude as the domains on the surface, whereas typical sizes of vesicles could be two to three orders of magnitude larger than the membrane thickness. Since shape changes in biological membranes are accompanied by changes in the transmembrane potential, it is useful to examine whether changes in the charge density on the surface could alter the curvature moduli of the membrane.

There have been many studies on the effect of surface charges on the curvature moduli of membranes. Winterhalter and Helfrich [6] and Lekkerkerker [7] determined the correction to the moduli due to adsorbed charges, and found that there is an increase in the mean curvature due to adsorbed charges, while the Gaussian curvature is negative. They also found a nonzero value for the spontaneous curvature when the charge densities on the two monolayers which form the bilayer are different. Kumaran [8] analyzed the distribution of sizes of vesicles formed due to the difference in charge density of asymmetrically charged membranes. The subsequent studies of Oberdisse and Portes $[9,10]$ also found that there is an increase in the elasticity due to adsorbed charges. Kumaran [11,12] also studied the thermodynamic and dynamical instability of a flat charged surface due to the motion of charges on the surface. In all these studies, the change in the electrostatic energy due to the curvature of the membrane is determined, and the corrections to the elasticity moduli are calculated from the energy change. The corrections to the elastic moduli are manifested as additional contributions to the curvature energy when a net curvature is imposed on the membrane. However, most of these studies used a linearization approximation (Debye-Huckel approximation) for the Poisson-Boltzmann equation, and therefore these studies are valid only at low charge densities. Consequently, they are not valid in realistic situations where the charge densities may be large. In the present analysis, the contributions to the curvature moduli due to electrostatic effects are calculated using the nonlinear Poisson-Boltzmann equation, thereby providing a complete solution for arbitrary charge densities.

The effect of charges on the dynamics of lipid bilayers has also been studied experimentally. Most studies focus on 
the importance of electric fields on the stability of membranes, and the electric fields necessary to cause rupture in membranes. These are studied using electroporation techniques, where the effect of electric fields on the stability of the membrane is assessed. It has been known for a long time [13] that application of a transverse electric field causes breakdown of a membrane, and the voltage necessary to cause rupture is termed the breakdown voltage. The process of rupture is not completely understood, and it was previously thought that the Maxwell stresses caused by the electric field at the surface, which acts like a mechanical stress, could cause rupture. Though the equivalence of the Maxwell stresses and mechanical stress on giant vesicles has been shown, it has been found that the tension at the breakdown voltage is too small to cause rupture [15]. Recent studies have indicated that the electric potential reduces the edge energy required to create a defect [14]. This is supported by theoretical studies [16] which show that the breakdown voltage depends not only on the charge density on the surface and the electrostatic repulsion between the head groups, but also on the type of hydrophobic groups that are incorporated in the lipid molecules. Further, electric effects are far fewer than those predicted by the Poisson-Boltzmann theory, and charge interactions could play a significant role in determining the dynamics of the membrane.

The present analysis provides some interesting results regarding the change in the curvature moduli due to surface charges. The electric fields are created by charges adsorbed or fixed on the membrane, and are far lower than those required for rupture. Though previous studies have indicated that the Poisson-Boltzmann approximation may not be numerically accurate, we expect the trends predicted here to be observed in real systems. Following earlier studies $[6,8]$, we assume that the charges on the two sides of the lipid bilayer are decoupled. Though this is not strictly true, it is a good first approximation when the dielectric constant of the hydrophobic groups is small compared to that of water. First, we consider charges that are fixed to the surface, so that the only contribution to the free energy is the electrostatic energy for assembling the charged layer. In the limit of low charge densities, the previous results of Winterhalter and Helfrich [6] for the curvature moduli and spontaneous curvature are recovered. However, in the limit of high charge densities, it is observed that the corrections to the mean and Gaussian curvature tend to a maximum value. The correction to the mean curvature is positive, while the correction to the Gaussian curvature is negative. In the limit of high charge density, it is found that the total curvature energy becomes negative, indicating that there is the spontaneous formation of vesicles when the charge density is increased beyond a critical value. This possibility was raised by Winterhalter and Helfrich [6] in their calculation of the curvature moduli using the DebyeHuckel approximation, though the parameter regime in which this transition takes place is outside the limits of validity of their analysis. An asymmetry in the charge density of the two monolayers that form the bilayer results in a spontaneous curvature, and the radius of curvature is large compared to the Debye length when the asymmetry is small. Calculations with realistic parameter values indicate that the radius of curvature could be of the order of microns when the Debye length is of the order of $10 \mathrm{~nm}$.

Another case considered in the present analysis is the effect of adsorbed charges on the curvature of the membrane. When charges are adsorbed, there is a reduction in the chemical energy due to adsorption, and the change in chemical energy is opposite in sign to the electrostatic energy. It is assumed that the energy of adsorption is independent of surface coverage, so that the reduction in chemical energy for every charge adsorbed is equal to the increase in the electrostatic energy of the last adsorbed charge. In this case, the change in the mean and Gaussian curvature at low charge densities is equal in magnitude and opposite in sign to that for fixed charges at low charge densities. However, at high charge densities, the mean and Gaussian curvatures for a membrane with adsorbed charges are identical to that for a membrane with fixed charges. The spontaneous curvature is opposite in sign to that for a surface with fixed charges at low charge density.

The electrostatic energy is calculated using the nonlinear Poisson-Boltzmann formalism in the next section. An expansion is used in a small parameter $\epsilon$, which is the ratio of the Debye length and the radius of curvature, and the electrostatic energy is determined by retaining terms correct to $O\left(\epsilon^{2}\right)$ in the expansion. The correction to the mean and Gaussian curvature moduli as well as the spontaneous curvature for a surface with fixed charges is given in Sec. III, while that for a surface with adsorbed charges is provided in Sec. IV. A brief summary of the important results is provided in Sec. V.

\section{ELECTROSTATIC ENERGY}

The electrostatic energy for a charged surface with charges on one side is first determined as a function of the curvature in the limit where the radius of curvature is large compared to the thickness of the counterion layer at the surface. The counterion density at the surface is determined using the Poisson-Boltzmann equations

$$
\begin{gathered}
\nabla^{2} \psi=-\left(n_{+}-n_{-}\right) / 2, \\
n_{+}=\exp (-\psi), \\
n_{-}=\exp (\psi),
\end{gathered}
$$

where $\psi$ is the scaled potential $\left(z e \psi^{*} / T\right), n_{+}=\left(n_{+}^{*} / N_{\infty}\right)$ and $n_{-}=\left(n_{-}^{*} / N_{\infty}\right)$ are the concentrations of the positively and negatively charged ions scaled by the ion concentration at a large distance from the surface $N_{\infty}$, and the scaled displacement $\mathbf{x}=\kappa \mathbf{x}^{*}$, where $\kappa$ is the inverse of the Debye screening length $\kappa=\left(2 N_{\infty} z^{2} e^{2} / \epsilon T\right)^{1 / 2}$. Here $\psi^{*}$ and $\mathbf{x}^{*}$ are the dimensional potential and distance coordinates, $z$ is the coordination number (assumed to be equal for the positive and negative ions), $e$ is the charge of an electron, $N_{\infty}$ is the ion concentration at a large distance from the surface, $\epsilon$ is the dielectric permittivity of the medium, and $T$ is the product of the Boltzmann constant and the temperature. The charge density at the surface per unit area $\sigma$ is determined from the requirement of electroneutrality, 


$$
\int d A \sigma=-\int d V\left(n_{+}-n_{-}\right),
$$

where the surface charge density $\sigma$ is scaled by $\left(N_{\infty} / \kappa\right)$. The relationship between the charge density and the potential can be used to simplify the above equation,

$$
\int d A \sigma=2 \int d V \nabla^{2} \psi=-2 \int d A \mathbf{n} \cdot \nabla \psi,
$$

where $d A$ is a differential area element on the charged surface, $\mathbf{n}$ is the unit normal at the surface directed into the solvent, while the integral over the surface at infinity is neglected since the difference in charge densities decays exponentially at large distances from the surface. If the charge density and potential are uniform at the surface, the electroneutrality condition reduces to

$$
\sigma=-2 \mathbf{n} \cdot \boldsymbol{\nabla} \psi,
$$

where $\mathbf{n}$ is the unit normal to the surface directed into the counterion layer, and there is a negative sign in the above equation because $\mathbf{n}$ is opposite in direction to the outward unit normal to the counterion layer.

The Poisson-Boltzmann equations (1) and (3) can be reduced to

$$
\nabla^{2} \psi=\sinh (\psi) .
$$

These are solved in cylindrical and spherical coordinates using a perturbation expansion in the parameter $\epsilon=(\kappa R)^{-1}$, where $R$ is the radius of curvature of the cylindrical or spherical surface. The solution for the cylindrical coordinate system is presented in detail, but only the results for the spherical coordinate system are presented since the methods of solution are identical for the two cases. It is useful to expand the radial coordinate in the curvilinear coordinate system as $r^{*}=R+\kappa^{-1} y$, where $R$ is the radial position of the surface and $y$ is the nondimensional distance from the surface. The scaled radial coordinate $r=\kappa r^{*}$ is then given by $r=\epsilon^{-1}+y$. The electrical potential is also expanded in a Taylor series in the parameter $\epsilon$,

$$
\psi=\psi_{0}+\epsilon \psi_{1}+\epsilon^{2} \psi_{2} .
$$

The Poisson-Boltzmann equation in a cylindrical coordinate system with variations only in the radial direction,

$$
\frac{1}{r} \frac{d}{d r} r \frac{d \psi}{d r}=\sinh (\psi),
$$

is expanded in the parameter $\epsilon$ to provide a hierarchy of equations for the corrections to the potentials,

$$
\begin{gathered}
\frac{d^{2} \psi_{0}}{d y^{2}}=\sinh \left(\psi_{0}\right) \\
\frac{d^{2} \psi_{1}}{d y^{2}}+\frac{d \psi_{0}}{d y}=\psi_{1} \cosh \left(\psi_{0}\right) \\
\frac{d^{2} \psi_{2}}{d y^{2}}+\frac{d \psi_{1}}{d y}-y \frac{d \psi_{0}}{d y}=\psi_{2} \cosh \left(\psi_{0}\right)+\left(\psi_{1}^{2} / 2\right) \sinh \left(\psi_{0}\right)
\end{gathered}
$$

The leading-order equation for $\psi_{0}$ is identical to that for the potential at a flat interface, and an analytical solution can be obtained for this case,

$$
\tanh \left(\psi_{0} / 4\right)=\tanh \left(\psi_{s} / 4\right) \exp (-y),
$$

where $\psi_{s}$ is the surface potential. The higher-order corrections to the potential cannot be evaluated analytically, but ordinary differential equations can be obtained for these corrections. It is convenient to effect a coordinate transform $u$ $=c \exp (-y)$, and define the constant $c=\tanh \left(\psi_{s} / 4\right)$. The domain of integration $0<y<\infty$ is now transformed to $c>u$ $>0$, and the equations for the corrections to the potential are

$$
\begin{gathered}
\left(u^{2} \frac{d^{2}}{d u^{2}}+u \frac{d}{d u}\right) \psi_{1}-g_{2}(u)=\psi_{1} g_{1}(u), \\
\left(u^{2} \frac{d^{2}}{d u^{2}}+u \frac{d}{d u}\right) \psi_{2}-u \frac{d \psi_{1}}{d u}+\ln (u / c) g_{2}(u) \\
=\psi_{2} g_{1}(u)+\frac{\psi_{1}^{2}}{2} g_{3}(u),
\end{gathered}
$$

where

$$
\begin{gathered}
g_{1}(u)=\left(\frac{8}{\left(1-u^{2}\right)^{2}}-\frac{8}{1-u^{2}}+1\right), \\
g_{2}(u)=\frac{4 u}{1-u^{2}}, \\
g_{3}(u)=\left(\frac{8 u^{3}}{\left(1-u^{2}\right)^{2}}+\frac{4 u}{1-u^{2}}\right) .
\end{gathered}
$$

The corrections to the potential can be separated into a homogeneous solution and a particular solution, $\psi_{1}=C_{1} \psi_{g}$ $+\psi_{p 1}$ and $\psi_{2}=C_{2} \psi_{g}+\psi_{p 2}$, where the homogeneous solution is determined from the equation

$$
\left(u^{2} \frac{d^{2}}{d u^{2}}+u \frac{d}{d u}\right) \psi_{g}-\psi_{g} g_{1}(u)=0
$$

and the particular solutions are determined from the inhomogeneous equations (12) and (13). The constants $C_{1}$ and $C_{2}$ are adjusted so that $\psi_{1}=\psi_{2}=0$ at $u=c(y=0)$. The above equations are solved numerically, and the values of the gradients of the potentials are obtained at the surface $y=0$. The numerical results show that subject to errors of the order of the machine accuracy, the equations for the gradients in the potentials at the surface are

$$
\begin{gathered}
\left.\frac{d \psi_{0}}{d y}\right|_{y=0}=\frac{-4 c}{1-c^{2}}, \\
\left.\frac{d \psi_{1}}{d y}\right|_{y=0}=-2 c, \\
\left.\frac{d \psi_{2}}{d y}\right|_{y=0}=\frac{c\left(1-c^{2}\right)}{2} .
\end{gathered}
$$


In the limit $\psi_{s} \rightarrow 0(c \rightarrow 0)$, the above solutions for the correction to the potential are

$$
\begin{aligned}
& \left.\frac{d \psi_{0}}{d y}\right|_{y=0}=-\psi_{s}, \\
& \left.\frac{d \psi_{1}}{d y}\right|_{y=0}=-\frac{\psi_{s}}{2}, \\
& \left.\frac{d \psi_{2}}{d y}\right|_{y=0}=\frac{\psi_{s}}{8} .
\end{aligned}
$$

These are consistent, up to $O\left(\epsilon^{2}\right)$, with the results obtained from the Debye-Huckel approximation for the potential outside a cylinder,

$$
\psi=\psi_{s}\left(\frac{K_{0}\left(\epsilon^{-1}+y\right)}{K_{0}\left(\epsilon^{-1}\right)}\right),
$$

where $J_{K}$ is the Bessel function. Here, we have substituted $r=\epsilon^{-1}+y$ consistent with the nondimensionalizations used.

A similar calculation can be carried out for a spherical surface, where the Laplace operator [equivalent to Eq. (9)] is

$$
\frac{1}{r^{2}} \frac{d}{d r} r^{2} \frac{d \psi}{d r}=\sinh (\psi)
$$

In this case, the results for the gradients in the potential are

$$
\begin{aligned}
& \left.\frac{d \psi_{0}}{d y}\right|_{y=0}=\frac{-4 c}{1-c^{2}}, \\
& \left.\frac{d \psi_{1}}{d y}\right|_{y=0}=-4 c .
\end{aligned}
$$

It was not possible to derive an exact analytical expression for the second correction to the potential gradient. However, an approximate form for the second correction is given by

$$
\left.\frac{d \psi_{2}}{d y}\right|_{y=0}=-c^{3} \sqrt{1-c^{2}}
$$

Correct to linear order in $\psi_{s}$ in the limit $\psi_{s} \rightarrow 0$, the corrections to the gradients of the potential at the surface are

$$
\begin{gathered}
\left.\frac{d \psi_{0}}{d y}\right|_{y=0}=-4 \psi_{s}, \\
\left.\frac{d \psi_{1}}{d y}\right|_{y=0}=-4 \psi_{s}, \\
\left.\frac{d \psi_{2}}{d y}\right|_{y=0}=0 .
\end{gathered}
$$

These are also consistent with the results obtained using the Debye-Huckel approximation for a spherical surface, where the potential is given by

$$
\psi=\psi_{s}\left(\frac{\exp (-y)}{\epsilon\left(\epsilon^{-1}+y\right)}\right)
$$

For the general case where the two principal radii of curvature are not equal, the governing equation for the potential is of the following form:

$$
\frac{d^{2} \psi}{d y^{* 2}}+\left(\frac{1}{R_{1}+y^{*}}+\frac{1}{R_{2}+y^{*}}\right) \frac{d \psi}{d y^{*}}=\kappa^{2} \sinh (\psi),
$$

where $y^{*}$ is the dimensional distance from the surface, $\kappa$ is the inverse of the Debye screening length, and $R_{1}$ and $R_{2}$ are the principal radii of curvature (it is assumed, without loss of generality, that $\left|R_{1}\right|<\left|R_{2}\right|$, and $R_{1}$ and $R_{2}$ are positive if the coordinate $y$ is along the outward unit normal direction joining the center of curvature to the point on the membrane surface). With the definitions $\epsilon=\left(\kappa R_{1}\right)^{-1}$ and $a_{r}$ $=\left(R_{1} / R_{2}\right)$, the governing equation for the potential becomes

$$
\frac{d^{2} \psi}{d y}+\left(\epsilon\left(1+a_{r}\right)-y \epsilon^{2}\left(1+a_{r}^{2}\right) \frac{d \psi}{d y}\right)=\sinh (\psi) .
$$

The results for the leading order and first correction to the potential gradient in this case are

$$
\begin{aligned}
& \left.\frac{d \psi_{0}}{d y}\right|_{y=0}=\frac{-2\left(1+a_{r}\right) c}{1-c^{2}}, \\
& \left.\frac{d \psi_{1}}{d y}\right|_{y=0}=-2\left(1+a_{r}\right) c .
\end{aligned}
$$

It was not possible to derive an exact analytical expression for the second correction to the potential gradient. However, an approximate form for the second correction is given by

$$
\left.\frac{d \psi_{2}}{d y}\right|_{y=0}=-a_{r} c^{3} \sqrt{1-c^{2}}+\frac{\left(1-a_{r}\right)^{2}}{2} c\left(1-c^{2}\right) .
$$

A comparison between the numerically calculated value of the second correction to the potential gradient and the value given by Eq. (27) is shown in Fig. 1.

The charge density at the surface can be calculated from the electroneutrality condition (6),

$$
\begin{aligned}
\sigma= & -\left.2\left(\frac{d \psi_{0}}{d y}+\epsilon \frac{d \psi_{1}}{d y}+\epsilon^{2} \frac{d \psi_{2}}{d y}\right)\right|_{y=0} \\
= & \frac{8 c}{1-c^{2}}+4 \epsilon\left(1+a_{r}\right) c+2 a_{r} c^{3} \sqrt{1-c^{2}} \\
& -\left(1-a_{r}\right)^{2} c\left(1-c^{2}\right) .
\end{aligned}
$$

Since the corrections to the potential in the above equation are functions of the coefficient $c=\tanh \left(\psi_{s} / 4\right)$, the surface 


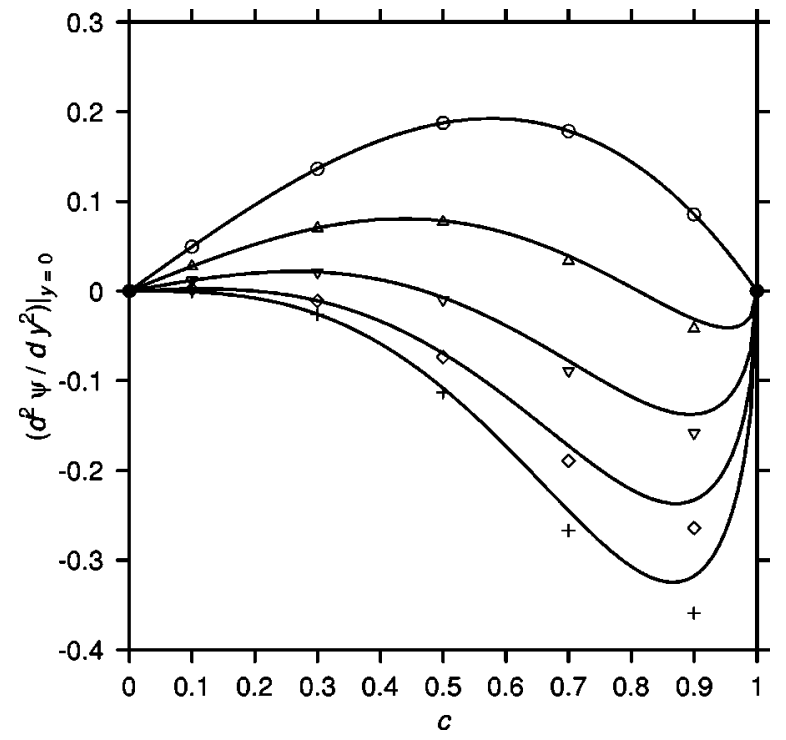

FIG. 1. Comparison of the numerical (symbols) and theoretical (lines) results for the second correction to the potential gradient at the surface $\left.\left(d \psi_{2} / d y\right)\right|_{y=0}$ as a function of the parameter $c$. The theoretical curves are obtained using Eq. (27). $\bigcirc, a_{r}=0 ; \triangle, a_{r}$ $=0.25 ; \nabla, a_{r}=0.5 ; \diamond, a_{r}=0.75 ;+, a_{r}=1.0$.

charge density is also obtained as a function of this parameter. Equations (26), (27), and (28) can be inverted to obtain $c$ as a function of the surface charge $\sigma$,

$$
c=c_{0}+\epsilon c_{1}+\epsilon^{2} c_{2},
$$

where

$$
\begin{gathered}
c_{0}=\frac{-4+\sqrt{16+\sigma^{2}}}{\sigma}, \\
c_{1}=-\frac{\left(1+a_{r}\right) c_{0}\left(1-c_{0}^{2}\right)^{2}}{2\left(1+c_{0}^{2}\right)}, \\
c_{2}=\frac{c_{0}\left(1-c_{0}^{2}\right)^{2}}{8\left(1+c_{0}^{2}\right)^{3}}\left[h_{1}\left(c_{0}\right)\left(1+a_{r}\right)^{2}+a_{r} h_{2}\left(c_{0}\right)\right],
\end{gathered}
$$

where

$$
\begin{gathered}
h_{1}\left(c_{0}\right)=\left(1-c_{0}^{2}\right)\left(3-4 c_{0}^{2}-3 c_{0}^{4}\right), \\
h_{2}\left(c_{0}\right)=2\left(1+c_{0}^{2}\right)^{2}\left(2 c_{0}^{2}-2-c_{0}^{2} \sqrt{1-c_{0}^{2}}\right) .
\end{gathered}
$$

The total electrostatic energy is determined from the energy required to assemble the charges at the surface in a potential field generated by the counterions in equilibrium,

$$
E^{e}=\int_{0}^{\sigma} \psi_{s}^{*} d \sigma^{*}
$$

where $\sigma^{*}$ is the variable of integration and $\psi_{s}^{*}$ is the surface potential when the surface charge density is $\sigma^{*}$. Analytical expressions for the electrostatic energy are obtained as a function of the surface potential,

$$
E^{e}=E_{0}^{e}+\epsilon E_{1}^{e}\left(1+a_{r}\right)+\epsilon^{2}\left[\left(1+a_{r}\right)^{2} E_{2}^{e^{\prime}}+a_{r} E_{2}^{e^{\prime \prime}}\right],
$$

where

$$
\begin{gathered}
E_{0}^{e}=\frac{32 c_{0} \operatorname{arctanh}\left(c_{0}\right)}{1-c_{0}^{2}}-\frac{16 c_{0}^{2}}{1-c_{0}^{2}}, \\
E_{1}^{e}=8 \ln \left(1-c_{0}^{2}\right), \\
E_{2}^{e^{\prime}}=-2 c_{0}^{2}+\frac{8 c_{0}^{2}}{1+c_{0}^{2}}, \\
E_{2}^{e^{\prime \prime}}=-8 c_{0}^{2}-\frac{16}{3}+\frac{8}{3} \sqrt{1-c_{0}^{2}}\left(c_{0}^{2}+2\right),
\end{gathered}
$$

where $c_{0}$ is related to $\sigma$ by Eq. (31).

\section{FIXED CHARGES}

In this section, the curvature moduli and the spontaneous curvature are determined for a membrane with different charge densities on the two sides, $\sigma_{a}$ and $\sigma_{b}$. Without loss of generality, $\sigma_{a}$ is considered to be the charge density on the monolayer that is stretched by the curvature, while $\sigma_{b}$ is on the monolayer compressed by the curvature. The charges are considered to be "fixed," in contrast to the analysis of the following section, where the charges are adsorbed from the solution adjoining the membrane. In this case, the total free energy has only an electrostatic contribution,

$$
\begin{aligned}
E^{e}= & {\left[E_{0}^{e}\left(\sigma_{a}\right)+E_{0}^{e}\left(\sigma_{b}\right)\right]+\epsilon\left(1+a_{r}\right)\left[E_{1}^{e}\left(\sigma_{a}\right)-E_{1}^{e}\left(\sigma_{b}\right)\right] } \\
& +\epsilon^{2}\left(1+a_{r}^{2}\right)\left[E_{2}^{e^{\prime}}\left(\sigma_{a}\right)+E_{2}^{e^{\prime}}\left(\sigma_{b}\right)\right] \\
& +\epsilon^{2} a_{r}\left[E_{2}^{e^{\prime \prime}}\left(\sigma_{a}\right)+E_{2}^{e^{\prime \prime}}\left(\sigma_{b}\right)\right] .
\end{aligned}
$$

Note that there is a negative sign in the coefficient of $\sigma_{b}$, because this surface is compressed by the curvature, and the unit normal to this surface is directed towards the center of curvature. In addition, we consider the limit where the difference in charge densities $\Delta \sigma=\left(\sigma_{a}-\sigma_{b}\right) \ll \sigma$, where $\sigma$ $=\left(\sigma_{a}+\sigma_{b}\right) / 2$. This is required because we have assumed that the spontaneous curvature is large compared to the Debye length, and this assumption is valid only in this limit. The electrostatic energy in this case, correct to quadratic order in $\epsilon$ and $\Delta \sigma$, is

$$
\begin{aligned}
E^{e}= & 2 E_{0}^{e}(\sigma)+\epsilon \Delta \sigma\left(1+a_{r}\right) \frac{d E_{1}^{e}}{d \sigma}+\epsilon^{2}\left(1+a_{r}^{2}\right)\left[2 E_{2}^{e^{\prime}}(\sigma)\right] \\
& +\epsilon^{2} a_{r}\left[2 E_{2}^{e^{\prime \prime}}(\sigma)\right] .
\end{aligned}
$$

If the above free energy is expressed in terms of the mean and Gaussian curvatures

$$
E^{e}=\frac{K_{m}^{e}}{2}\left(1+a_{r}-K_{0}^{e}\right)^{2} \epsilon^{2}+K_{g}^{e} a_{r} \epsilon^{2}+E_{0}^{e^{\prime}}(\sigma),
$$


the mean and Gaussian curvatures, as well as the spontaneous curvature, are easily deduced from the above corrections to the free energy,

$$
\begin{gathered}
K_{m}^{e}=4 E_{2}^{e^{\prime}}(\sigma), \\
K_{g}^{e}=2 E_{2}^{e^{\prime \prime}}(\sigma), \\
K_{0}^{e}=-\frac{1}{4 E_{2}^{e^{\prime}}} \frac{d E_{1}^{e}}{d \sigma} \Delta \sigma .
\end{gathered}
$$

In the Debye-Huckel approximation $\sigma \rightarrow 0$, the above curvatures have the following limiting form:

$$
\begin{gathered}
K_{m}^{e}=\left(3 \sigma^{2} / 8\right), \\
K_{g}^{e}=-\left(\sigma^{2} / 4\right), \\
K_{0}^{e}=(2 \Delta \sigma / 3 \sigma) .
\end{gathered}
$$

The above solutions are identical to those derived by Winterhalter and Helfrich for weakly charged membranes, for the nondimensionalizations used here. There is an apparent difference of a factor of 2 in the equation for the spontaneous curvature, because Winterhalter and Helfrich have defined $\sigma_{a}=\sigma(1+\delta)$ and $\sigma_{b}=\sigma(1-\delta)$, while we have defined $\sigma_{a}$ $=(\sigma+\Delta \sigma / 2)$ and $\sigma_{b}=(\sigma-\Delta \sigma / 2)$. In addition, the positive constant in the equation for the spontaneous curvature indicates that $K_{0}^{e}>0$ for $\Delta \sigma>0$, or the higher charge density is on the monolayer stretched by the curvature. It is also useful to examine the curvatures in the limit of high charge densities $\sigma \gg 1$. In this limit, the curvatures have the following limiting forms:

$$
\begin{gathered}
K_{m}^{e}=8-\frac{256}{\sigma^{2}}, \\
K_{g}^{e}=-\frac{80}{3}+\frac{32 \sqrt{2}}{\sqrt{\sigma}}+\frac{128}{\sigma}, \\
K_{0}^{e}=\frac{\Delta \sigma}{\sigma}\left(1-\frac{4}{\sigma}\right) .
\end{gathered}
$$

It is interesting to note that the Gaussian curvature is negative, indicating that the Gaussian curvature contribution to the electrostatic energy tends to favor the formation of surfaces whose principal curvatures have the same sign. The behavior of the mean and Gaussian curvature energies and the spontaneous curvature in the intermediate regime are shown in Fig. 2. It is observed that the mean curvature is positive, and increases to its maximum value of 8 at large charge density. The Gaussian curvature is negative, and decreases as the charge density is increased. The spontaneous curvature varies in a narrow range from $(2 \Delta \sigma / 3 \sigma)$ at low charge density to $(\Delta \sigma / \sigma)$ at high charge density.

The limiting forms (39) and (40) also raise the interesting possibility that there could be spontaneous formation of

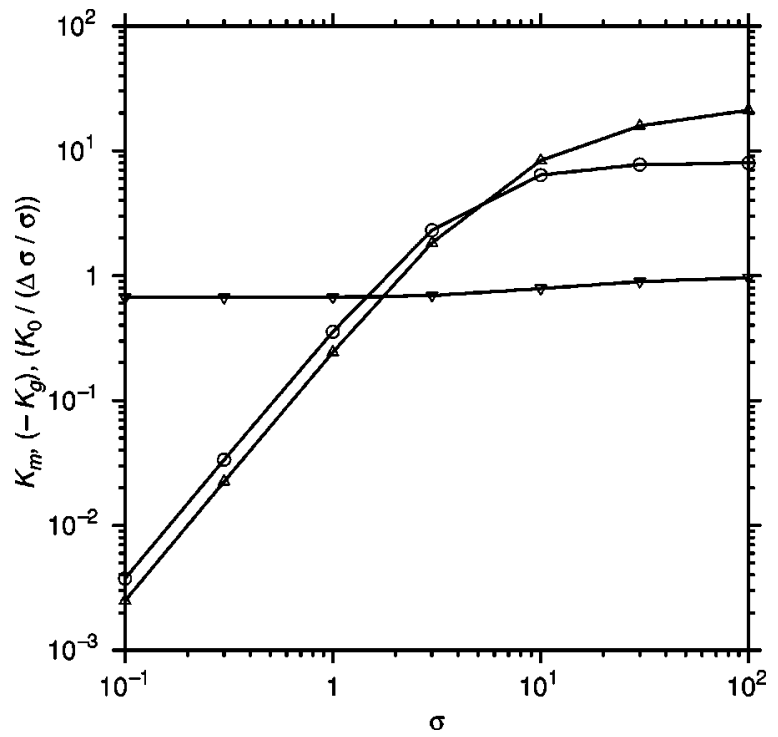

FIG. 2. The mean curvature $K_{m}^{e}(\bigcirc)$, Gaussian curvature $K_{g}^{e}$ $(\triangle)$, and spontaneous curvature $K_{0}^{e}(\nabla)$ as a function of $\sigma$ for a surface with fixed charges.

vesicles if the surface charge density increases beyond a critical value, even in the absence of charge asymmetry. In the absence of spontaneous curvature, the difference between the electrostatic free energy of a spherical vesicle and a flat surface of equal area is given by

$$
E^{e}=8 \pi K_{m}^{e}+4 \pi K_{g}^{e} .
$$

There is a spontaneous transition to a vesicular state if the above energy change is negative. It is observed from Eqs. (39) that the right side of the above equation is positive in the limit $\sigma \ll 1$, but Eqs. (40) show that the right side of Eq. (41) is negative for $\sigma \gg 1$. This indicates that there is a spontaneous transition from a flat state to a vesicular state at intermediate values of $\sigma$, and numerical calculations indicate that this transition takes place at $\sigma=28.25$. The energy per vesicle $\left(8 \pi K_{m}^{e}+4 \pi K_{g}^{e}\right)$ is shown as a function of $\sigma$ in Fig. 3 for the case of fixed charges. It is useful to examine whether this effect can be observed in real systems of interest. The dimensional charge density required for this transition is $\sigma^{*}=28.25 N_{\infty} / \kappa$, where $N_{\infty}$ is the molar density of the counterions at a large distance from the surface and $\kappa$ is the inverse of the Debye length. For $N_{\infty}=10^{-2} \mathrm{~mol} / \mathrm{l}$, the Debye screening length is about $3 \mathrm{~nm}$ and the surface charge density works out to $\sigma^{*} \sim 6 \times 10^{17} \mathrm{~m}^{-2}$. This is higher than the charge densities typically observed in biological membranes, but only by about an order of magnitude.

It is useful to estimate the magnitudes of the curvature energies due to electrostatic interactions, and determine whether these are likely to be of the same magnitude as those due to the intrinsic curvature of the membrane. The curvature energy per unit area scales as $\left(\sigma^{*} z e \psi^{*}\right)$, and therefore the Gaussian and mean curvatures scale as $\left(\sigma^{*} z e \psi^{*} / \kappa^{2}\right)$, since the Debye length $\kappa$ is the only length scale in the system. Using the scalings $\sigma^{*} \sim N_{\infty} / \kappa$ and $\psi^{*} \sim T / z e$, the curvatures scale as $N_{\infty} T / \kappa^{3}$, or $\left[\epsilon^{3 / 2} T^{5 / 2} /\left(N_{\infty}^{1 / 2} e^{3} z^{3}\right)\right]$. For an 


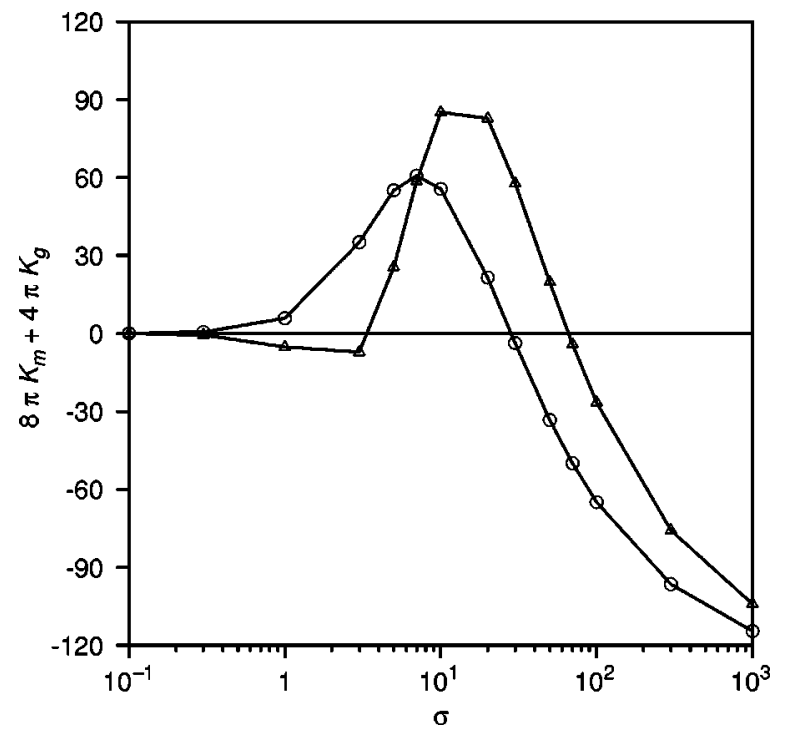

FIG. 3. The total energy of a vesicle $\left(8 \pi K_{m}+4 \pi K_{g}\right)$ as a function of $\sigma$ for a surface with fixed charges $(\bigcirc)$ and adsorbed charges $(\triangle)$.

electrolyte with coordination number $z=1$, this is $O(2$ $\times 10^{-22} / N_{\infty}^{1 / 2}$ ) J, where $N_{\infty}$ is the molar concentration in $\mathrm{mol} / \mathrm{l}$. Therefore, the curvature energy scale varies between $10^{-20} \mathrm{~J}$ for very low electrolyte concentrations of $0.1 \mathrm{mM}$ and $10^{-22} \mathrm{~J}$ for high electrolyte concentrations of $1 \mathrm{M}$. This is close to the bending energy of typical lipid bilayers of $10^{-19} \mathrm{~J}$ [8], indicating that the electrostatic contributions could be significant in determining the bending energy of the charged surface at low ion concentrations.

When there is charge asymmetry, the spontaneous curvature is proportional to $(\Delta \sigma / \sigma)$. This implies that the radius of curvature is large compared to the Debye screening length when the charge asymmetry $\Delta \sigma$ is small compared to the mean charge density $\sigma$. Therefore, spontaneous formation of vesicles with radius large compared to the Debye length would be observed when the charge asymmetry is small compared to the average charge density of the surface. For example, a difference in charge densities of $1 \%$ on the two sides of the membrane could give rise to micron-sized vesicles when the Debye screening length is $10 \mathrm{~nm}$.

In case the surface has an intrinsic mean curvature energy $K_{i}$, the total energy per unit area of the surface is

$$
E=\frac{K_{m}^{i}}{2}\left(\frac{1}{R_{1}}+\frac{1}{R_{2}}\right)^{2}+\frac{K_{m}^{e}}{2}\left(\frac{1}{R_{1}}+\frac{1}{R_{2}}-K_{0}^{e}\right)^{2}+\frac{K_{g}^{e}}{R_{1} R_{2}} .
$$

This results in modified mean and spontaneous curvatures,

$$
\begin{gathered}
K_{m}=\left(K_{m}^{i}+K_{m}^{e}\right), \\
K_{0}=K_{m}^{e} K_{0}^{e} /\left(K_{m}^{i}+K_{m}^{e}\right) .
\end{gathered}
$$

This has the effect of further reducing the spontaneous curvature of the membrane and increasing the radius of curvature. For $K_{m}^{e} / K_{m}^{i} \sim 0.01$ as indicated by the above calcula- tions, the spontaneous curvature could be of the order of $10 \mu \mathrm{m}$ when the Debye screening length is $1 \mathrm{~nm}$.

\section{ADSORBED CHARGES}

The adsorption of the charged species onto the surface results in a reduction in the chemical energy. Here, it is assumed that the adsorbed species on the surface is noninteracting, so that the reduction in chemical energy per adsorbed molecule is a constant. This would overestimate the concentration of the adsorbed species on the surface, because it disregards any enthalpic or electrostatic interaction between the charged species on the surface. Therefore, this provides an upper bound on the possible curvature due to the charge density-curvature coupling. However, we adopt this simplification in order to avoid dealing with specific adsorption models, which involve additional parameters.

When the adsorbed layer is in equilibrium with the bulk, the reduction in the chemical energy due to the last adsorbed charge is equal to the increase in the electrostatic energy of adsorption, which is $\psi_{s}$ when nondimensionalized by $(z e / T)$. If the charges are noninteracting so that the reduction in energy per charge is a constant, the total reduction in the electrostatic energy per unit area is $\sigma \psi_{s}$. The total energy per unit area, which is the sum of the electrostatic and chemical components, is given by

$$
E^{e c}=\int_{0}^{\sigma} \psi_{s}^{*}\left(\sigma^{*}\right) d \sigma^{*}-\psi_{s} \sigma
$$

Using the expressions derived in the preceding section, the following corrections to the free energy are obtained:

$$
E^{e c}=E_{0}^{e c}+\epsilon E_{1}^{e c}\left(1+a_{r}\right)+\epsilon^{2}\left[\left(1+a_{r}\right)^{2} E_{2}^{e c^{\prime}}+a_{r} E_{2}^{e c^{\prime \prime}}\right]
$$

where

$$
\begin{gathered}
E_{0}^{e c}=-\frac{16 c_{0}^{2}}{1-c_{0}^{2}}, \\
E_{1}^{e c}=8 \ln \left(1-c_{0}^{2}\right)+\frac{16 c_{0}^{2}}{1+c_{0}^{2}}, \\
E_{2}^{e c^{\prime}}=-\frac{2 c_{0}^{2}\left(3-15 c_{0}^{2}+c_{0}^{4}+3 c_{0}^{6}\right)}{\left(1+c_{0}^{2}\right)^{3}},
\end{gathered}
$$

$$
\begin{aligned}
E_{2}^{e c^{\prime \prime}=} & -\frac{8 c_{0}^{2}\left(3 c_{0}^{2}-1\right)}{1+c_{0}^{2}}-\frac{16}{3} \\
& +\frac{8}{3\left(1+c_{0}^{2}\right)} \sqrt{1-c_{0}^{2}}\left(2+3 c_{0}^{2}+4 c_{0}^{4}\right) .
\end{aligned}
$$

The mean and Gaussian curvature energies and the spontaneous curvature are calculated using relations similar to Eq. 
(42) for the surface with fixed charges. In the limit $\sigma \ll 1$, these curvature energies have the following limiting behavior:

$$
\begin{gathered}
K_{m}^{e c}=-\left(3 \sigma^{2} / 8\right), \\
K_{g}^{e c}=\left(\sigma^{2} / 4\right), \\
K_{0}^{e c}=(2 \Delta \sigma / 3 \sigma) .
\end{gathered}
$$

The mean and Gaussian curvature are negative of those for the case with fixed charges, while the spontaneous curvature remains unchanged. The reason for this is as follows. In the limit of small $\sigma$, the surface charge density $\psi_{s}$ is proportional to $\sigma$. Therefore, the electrostatic energy (46) is equal to $\left(\sigma \psi_{s} / 2\right)$, while the change in the chemical energy is $-\left(\sigma \psi_{s}\right)$. Consequently, the total energy for the adsorbed charges is the negative of the electrostatic energy. It is also observed that the mean curvature is negative for a membrane with adsorbed charges, and therefore the curvature modulus decreases due to the adsorption of the charges. The Gaussian curvature is positive, and tends to favor surfaces with principal curvatures of opposite sign. The spontaneous curvature remains unchanged. An interesting consequence of the curvature energies (46) and (47) is that the adsorption of charges favors the formation of vesicles, since the energy per unit area $\left(2 K_{m}^{e c}+K_{g}^{e c}\right)$ is negative in this case. However, membranes also have an intrinsic mean curvature $K_{m}^{i}$ which is positive, and therefore there is a spontaneous transition to vesicles only when $4 \pi\left(2 K_{m}^{i}+2 K_{m}^{e c}+K_{g}^{e c}\right)$ is negative, or for $\sigma>2\left(K_{m}^{i}\right)^{1 / 2}$. If we assume the dimensional intrinsic curvature of the membrane $K_{m}^{i}=10^{-19} \mathrm{~J}$, and the energy scale for the charged surface is $10^{-20} \mathrm{~J}$ as calculated above for weak electrolytes, the transition to vesicles occurs for $\sigma>6$. This is certainly outside the regime of validity of the linearization approximation, so this effect is not likely to generate a transition for weakly charged biological membranes. However, the present calculation indicates that the charged surface should have a curvature energy that is lower than the uncharged surface.

In the limit $\sigma \gg 1$, the limiting forms for the mean and Gaussian curvature are

$$
\begin{gathered}
K_{m}^{e c}=8-\frac{768}{\sigma^{2}}, \\
K_{g}^{e c}=-\frac{80}{3}+\frac{48 \sqrt{2}}{\sqrt{\sigma}}+\frac{256}{\sigma}, \\
K_{0}^{e c}=\frac{\Delta \sigma}{\sigma}\left(1-\frac{8}{\sigma}\right) .
\end{gathered}
$$

The leading-order solutions for the curvatures in this limit are identical to those for a surface with fixed charges. The behavior of the mean, Gaussian, and spontaneous curvature in the intermediate regime is shown in Fig. 4. It is observed that the mean curvature is negative at low charge densities, as predicted by Eqs. (46), and becomes positive at high

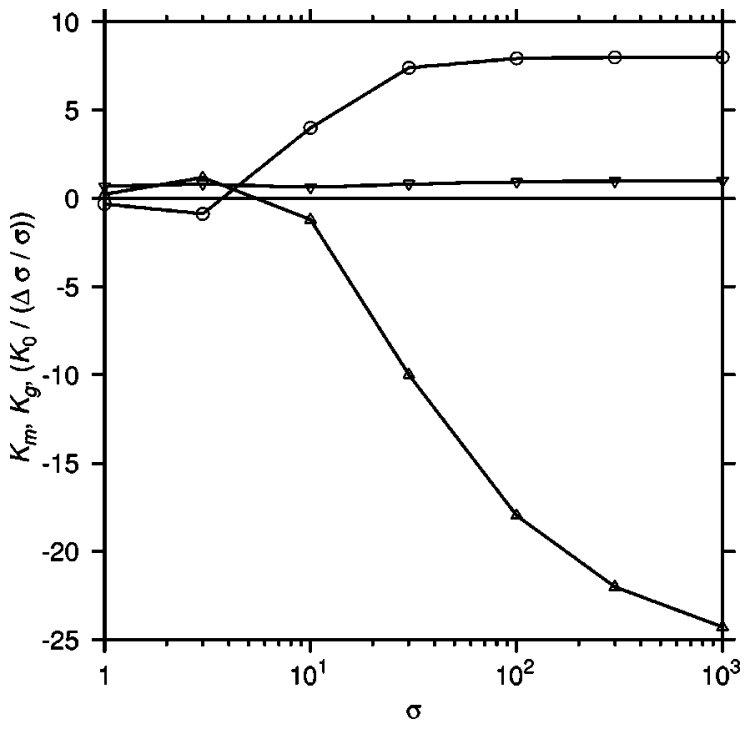

FIG. 4. The mean curvature $K_{m}^{e c}(\bigcirc)$, Gaussian curvature $K_{g}^{e c}$ $(\triangle)$, and spontaneous curvature $K_{0}^{e c}(\nabla)$ as a function of $\sigma$ for a surface with adsorbed charges.

charge densities as predicted by Eqs. (47). The Gaussian curvature has the opposite behavior, and goes from positive values at low charge density to negative values at high charge density. The spontaneous curvature has a behavior similar to that for a system with fixed charges. The variation of $\left(8 \pi K_{m}^{e c}+4 \pi K_{g}^{e c}\right)$ as a function of $\sigma$ is shown in Fig. 2 for a surface with adsorbed charges. It is observed that the curvature energy due to charge adsorption is negative both for small and large $\sigma$, and becomes positive at intermediate values of $\sigma$.

The spontaneous curvature in the present case requires careful interpretation, since the correction to the mean curvature due to electrostatic effects $K_{m}^{e c}$ is negative. If the intrinsic mean curvature $K_{m}^{i}$ is positive and larger in magnitude than $K_{m}^{e c}$, the effective spontaneous curvature of the surface is $K_{0}=K_{m}^{e c} K_{0}^{e c} /\left(K_{m}^{i}+K_{m}^{e c}\right)$. This is opposite in sign to $K_{0}^{e c}$, since the mean curvature modulus $K_{m}^{e c}$ is negative. Consequently, the mean curvature in the present case is opposite in sign to that for a system with fixed charges, and favors a higher charge density on the monolayer which is compressed by the curvature.

\section{CONCLUSIONS}

The change in the mean and Gaussian curvature moduli and the spontaneous curvature due to charges on a membrane were analyzed using the Poisson-Boltzmann equation. The linearization approximation was not made in the present analysis, so this calculation provides the coupling between the charge density and curvature even for strongly charged surfaces. Previous calculations, which used the linearized Debye-Huckel formalism, indicated that the mean and Gaussian curvatures increase as the square of the charge density at low charge density. These results are recovered here, but the present analysis also indicates that there is a saturation of the mean and Gaussian curvatures at high charge 
densities. The mean curvature saturates to a value of 8 [when scaled by $\left.\left(N_{\infty} T / \kappa^{3}\right)\right]$ at high charge densities, while the Gaussian curvature increases to a value of $-(40 / 3)$ in this limit. Thus the presence of charges increases the mean curvature modulus, and results in a negative Gaussian curvature modulus, which favors principal curvatures of equal signs. The magnitude of the curvature energy $\left(N_{\infty} T / \kappa^{3}\right)$ varies between $10^{-20} \mathrm{~J}$ and $10^{-22} \mathrm{~J}$ for variations in the $N_{\infty}$ between $0.1 \mathrm{mM}$ and $1 \mathrm{M}$. Therefore, the electrostatic curvature energies are close to the intrinsic curvature energy estimated to be $10^{-19} \mathrm{~J}[8]$ in the limit of high charge densities. This indicates that surface charges could have a significant effect on the curvature energies.

Another interesting possibility is the reduction in the total curvature energy in the limit of high charge densities. It was speculated by Winterhalter and Helfrich [6] that the total curvature energy $8 \pi K_{m}^{e}+4 \pi K_{g}^{e}$ may become negative at high charge densities, resulting in the spontaneous formation of vesicles when the curvature energy is due to electrostatic effects alone. However, they were not able to verify, since their calculations were based on the Debye-Huckel approximation, which is not valid for high charge densities. The present calculation indicates that such a transition does take place at $\sigma=28.5$, and the total curvature energy is negative at high charge densities even in the absence of asymmetry in the charge density.

The spontaneous curvature, scaled by the inverse of the Debye length, varies proportional to $(\Delta \sigma / \sigma)$, where $\Delta \sigma$ is the asymmetry in the charge density on the two sides of the membrane and $\sigma$ is the average charge density. The coefficient of proportionality varies in a small range between $2 / 3$ at low charge density and 1 at high charge density. The positive coefficient indicates that the energy is lowered when the monolayer with the higher charge density is stretched by the curvature. This raises the possibility of the spontaneous formation of vesicles of radius large compared to the Debye length when the charge asymmetry is small compared to the mean charge density of the membrane.

The coupling between charge density and curvature for a membrane with adsorbed charges was also considered in the limit where there is no enthalpic interaction between the charges on the surface, so that the gain in chemical energy per charge adsorbed is equal to the loss of electrostatic energy for the last charge adsorbed on the surface. It was found that the corrections to the mean and Gaussian curvatures in this case are opposite in sign to that for the case with fixed charges at low charge densities. However, at high charge densities, the mean and Gaussian curvature are identical to those for the case with fixed charges. The variation of the spontaneous curvature is similar to that for the case of fixed charges. The negative mean curvature at low charge densities for membranes with adsorbed charges indicates that electrostatic effects tend to reduce the curvature modulus, and favor spontaneous formation of vesicles. However, the magnitudes of the mean curvature are small compared to the intrinsic curvature, indicating that electrostatic effects are likely to soften the membrane in the limit of low charge densities, but are unlikely to cause spontaneous formation of vesicles. A charge asymmetry could cause a spontaneous curvature in which the radius of curvature is large compared to the Debye length in this case as well. However, in contrast to the case of fixed charges, the surface which is stretched by the curvature has a lower charge density, while the surface which is compressed has a higher charge density.
[1] E. W. Kaler, A. K. Murthy, B. E. Rodriguez, and J. A. N. Zasadzinski, Science 245, 1371 (1989).

[2] A. Voight and E. Donath, in Biophysics of the Cell Surface, edited by R. Glaser and D. Gingell (Springer-Verlag, Berlin, 1990).

[3] M. D. Houslay and K. K. Staney, Dynamics of Biological Membranes (Wiley, New York, 1982).

[4] R. Lipowsky and E. Sackmann, Structure and Dynamics of Membranes (Elsevier, Amsterdam, 1995).

[5] S. A. Safran, P. A. Pincus, D. Andelman, and F. C. MacKintosh, Phys. Rev. A 43, 1071 (1991).

[6] M. Winterhalter and W. Helfrich, J. Phys. Chem. 92, 6865 (1988)
[7] H. N. W. Lekkerkerker, Physica A 167, 384 (1990).

[8] V. Kumaran, J. Chem. Phys. 99, 5490 (1993).

[9] J. Oberdisse and G. Porte, Phys. Rev. E 56, 1965 (1997).

[10] J. Oberdisse, Eur. Phys. J. B 3, 463 (1998).

[11] V. Kumaran, Phys. Rev. Lett. 85, 4996 (2000).

[12] V. Kumaran, Phys. Rev. E 64, 011911 (2001).

[13] R. Benz, F. Beckers, and U. Zimmermann, J. Membr. Biol. 48, 181 (1979).

[14] R. W. Glaser et al., Biochim. Biophys. Acta 940, 275 (1988).

[15] D. Needham and R. S. Nunn, Biophys. J. 58, 997 (1990).

[16] A. Diedrich, G. Bahr, and M. Winterhalter, Phys. Rev. E 58, 4883 (1998). 